\title{
Rare occurrence of bilateral breast and peritoneal metastases from osteogenic sarcoma
}

\author{
Ruoh Shyuan $\underline{C h a n}^{1}$, MBBS, FRCR, Gnana $\underline{K u m a r}^{2}$, MBBS, FRCR, Anushya A/P $\underline{\text { Vijayananthan }}^{2}$, MBBS, MRad
}

\begin{abstract}
Breast metastases are uncommon and typically spread from contralateral breast carcinomas. Breast metastases that spread from extramammary malignancies are even less common, and account for $0.5 \%-6.6 \%$ of all malignant breast disease. As extrapulmonary metastases from osteosarcoma are uncommon, breast metastasis from osteosarcoma is extremely rare. We report a case of breast and peritoneal metastases from a tibial osteosarcoma 18 months after diagnosis, and 9 months after surgery and adjuvant chemotherapy. Computed tomography findings of multiple calcified and noncalcified tumour deposits in the lungs, pleura, peritoneum, chest wall and both breasts are described.
\end{abstract}

Keywords: breast metastases, cutaneous metastases, osteosarcoma, peritoneal metastases

\section{INTRODUCTION}

Metastasis to the breast is an uncommon occurrence, especially in metastatic osteosarcoma (osteogenic sarcoma). The most common site of metastases from an osteosarcoma is the lung, thus non-pulmonary metastases from osteosarcoma are less frequent. Metastases to soft tissue or abdominal organs are also very uncommon. ${ }^{(1)}$ Here, we report breast and peritoneal metastases from an osteosarcoma with description of the computed tomography $(\mathrm{CT})$ findings in a patient who developed pulmonary, pleural, peritoneal, cutaneous and breast metastases from a tibial osteosarcoma nine months after surgery and adjuvant chemotherapy.

\section{CASE REPORT}

A 16-year-old Chinese girl was diagnosed with right tibial osteosarcoma 18 months prior to her current presentation. She had undergone resection of the tumour, followed by insertion of an endoprosthesis and chemotherapy. Histopathological examination found $80 \%$ tumour necrosis with minimal local tumour invasion. Thoracic CT at the time of diagnosis was normal. A bone scan performed four months after the completion of treatment demonstrated increased radiotracer uptake at the inferior angle of the left scapula, corresponding to a lytic lesion visualised on radiographs, which was consistent with metastasis. However, follow-up thoracic CT remained normal, and she subsequently defaulted treatment.

Nine months later, the patient presented to a private gynaecologist with a one-week history of abdominal pain. Ultrasonography examination revealed the presence of a large central mesenteric mass. The uterus and ovaries were normal. A preliminary diagnosis of metastatic tumour was made and she was referred to our hospital for further assessment. Despite being slightly pale, the patient's vital signs were stable, and there was no tachypnoea. Multiple skin nodules less than $5 \mathrm{~cm}$ in size located in

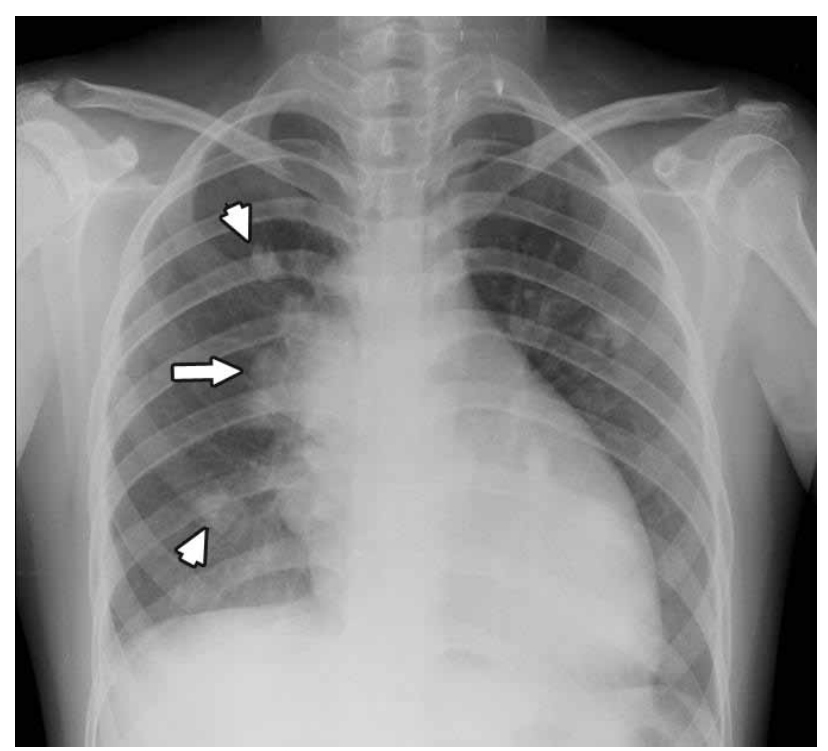

Fig. 1 Posteroanterior chest radiograph shows multiple lung metastases, some of which are calcified (arrowheads), and lobulated right perihilar lymph nodes (arrow).

the inframammary region of the anterior chest wall were seen. Palpation of the abdominal region revealed a mobile and slightly tender central abdominal mass of approximately $10 \mathrm{~cm} \times 16 \mathrm{~cm}$. The patient was slightly anaemic with a haemoglobin level of $83 \mathrm{~g} / \mathrm{L}$. There was borderline leucocytosis with a total white blood cell count of $11.4 \times 10^{9} / \mathrm{L}$, slight neutrophilia with a neutrophil count of $8.1 \times 10^{9} / \mathrm{L}$, and a lower normal limit platelet count of $150 \times 10^{9} / \mathrm{L}$.

Posteroanterior chest radiography demonstrated the presence of multiple lung nodules with right hilar lymphadenopathy (Fig. 1), which was consistent with lung and lymph node metastases. CT of the thorax and abdomen identified multiple lobulated, heterogeneously enhancing soft tissue masses with dense central calcifications in the lungs, along the perihilar mediastinal pleura and on the diaphragmatic pleura, with

${ }^{1}$ Department of Diagnostic Imaging, Hospital Kuala Lumpur, ${ }^{2}$ Department of Biomedical Imaging, University Malaya Medical Centre, Kuala Lumpur, Malaysia Correspondence: Dr Ruoh Shyuan Chan, Radiologist, Hospital Kuala Lumpur, Jalan Pahang, Kuala Lumpur 50586, Malaysia. ruohshyuan@gmail.com 


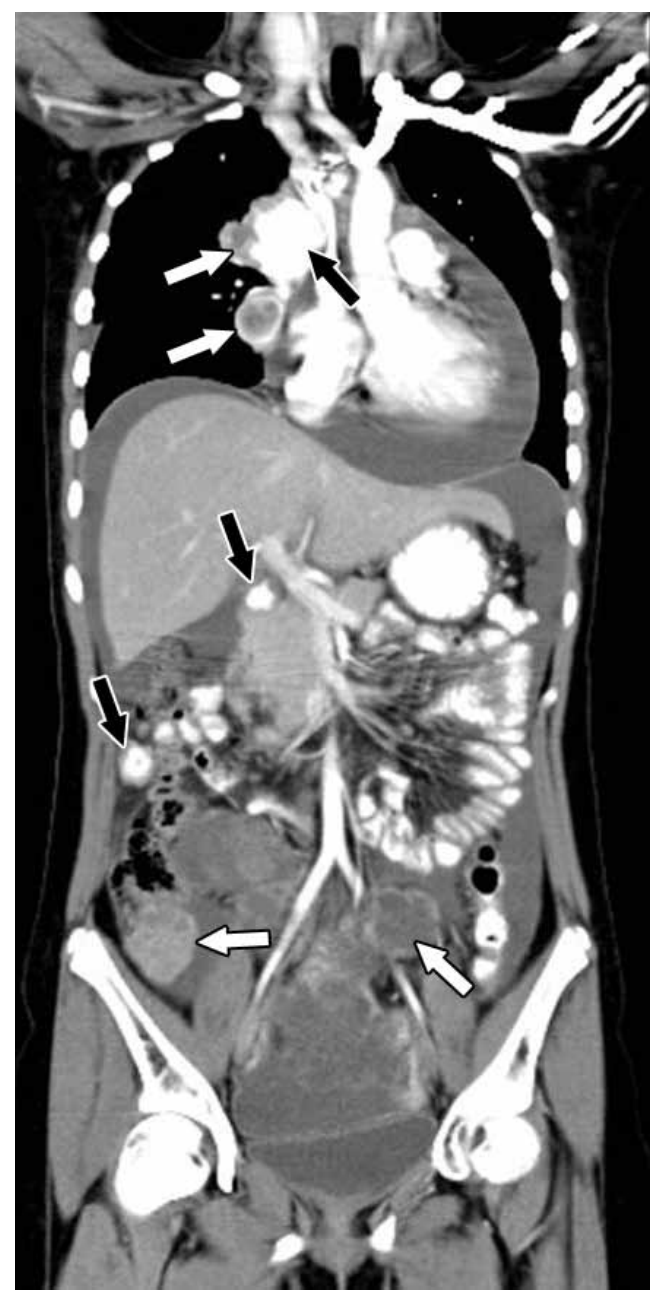

Fig. 2 Coronal reformatted CT image shows calcified metastatic deposits (black arrows) and noncalcified metastatic deposits (white arrow) at the right hilum and in the peritoneum.

associated pleural and pericardial effusions. Moderate ascites was present in the patient's abdomen, with soft tissue masses of similar appearance on the parietal peritoneum in the subphrenic, paracolic and peripancreatic regions. One of the peritoneal masses in the right paracolic region and another pulmonary mass in the left lung were noncalcified but homogeneously enhancing. There were also multiple large, lobulated, poorly enhancing and conglomerating intraperitoneal mesenteric soft tissue masses, which corresponded to the palpable central abdominal mass. Clear fat planes were seen between these masses and the abdominal viscera. The liver, spleen, pancreas, adrenals and kidneys were normal (Fig. 2). There were multiple discrete, round nodules in the breasts, with dense calcification seen in the larger nodules. Several smaller, round, calcified and noncalcified subcutaneous nodules were seen in the inframammary region of the anterior chest wall, with another paramedian nodule in the left lower abdominal wall (Figs. 3 \& 4).

The patient and her family were counselled on the poor prognosis of the disease, and her family was reluctant for her to undergo a second cycle of chemotherapy. They eventually decided not to pursue any further investigation or treatment in the hospital and sought alternative palliative treatment. She has been lost to follow-up since then.

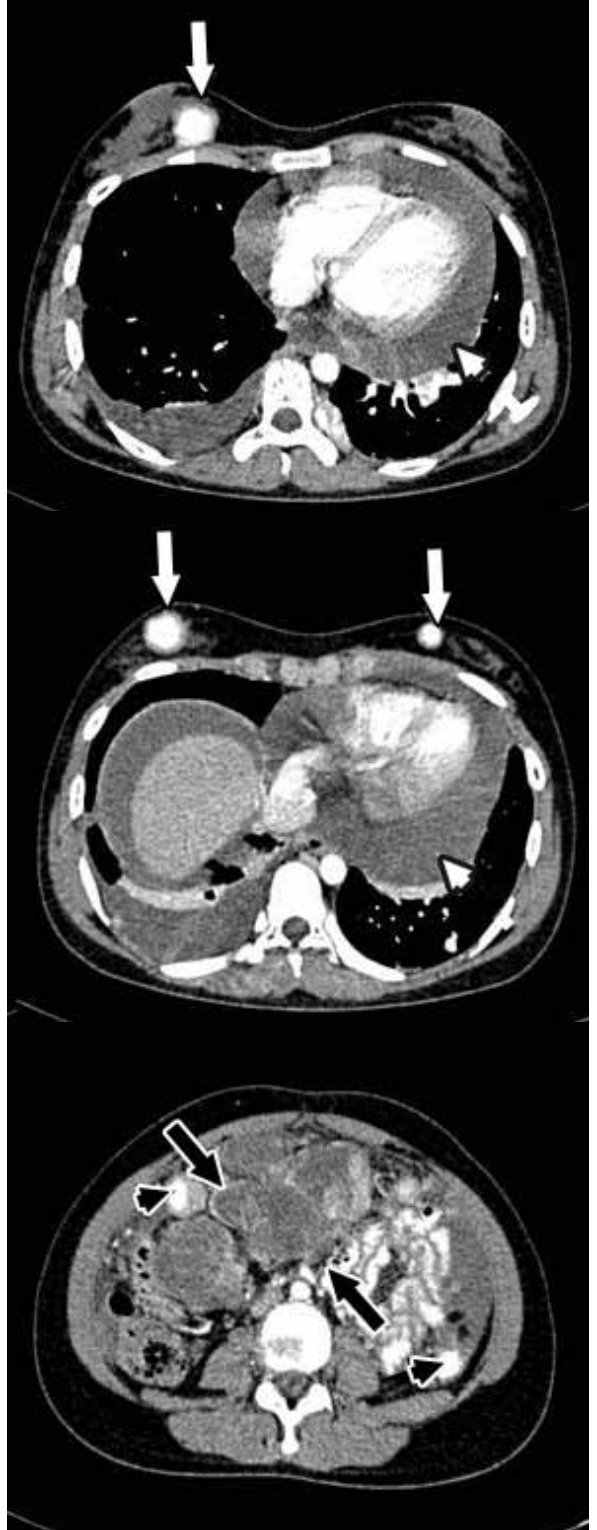

Fig. 3 Contrast-enhanced CT images of the thorax and abdomen show tumour deposits in both breasts (white arrows), peritoneum (black arrowhead) and mesentery (black arrows), with associated moderate pericardial effusion (white arrowheads), pleural effusion and ascites.

\section{DISCUSSION}

Osteosarcoma primarily affects adolescents and young adults, with the long bones of the extremities most commonly involved. The lungs are the most frequent (62\%) site of first recurrence, followed by local (20\%) and other distant (19\%) sites. ${ }^{(2)}$ As with most sarcomas, osteosarcomas initially metastasise haematogenously, most commonly to the lungs. ${ }^{(1)}$ However, according to Giuliano et al, there have been changes in the pattern of metastatic disease from osteosarcoma over the past two decades due to the advent of adjuvant chemotherapy, with a higher incidence of extrapulmonary metastases observed in their cohort of patients who underwent adjuvant chemotherapy, with or without concurrent pulmonary metastases. The authors also reported a larger proportion of patients who were disease-free at three years. ${ }^{(3)}$

Despite aggressive treatment, a large number of nonmetastatic, high-grade osteosarcoma of the extremities remains incurable, 


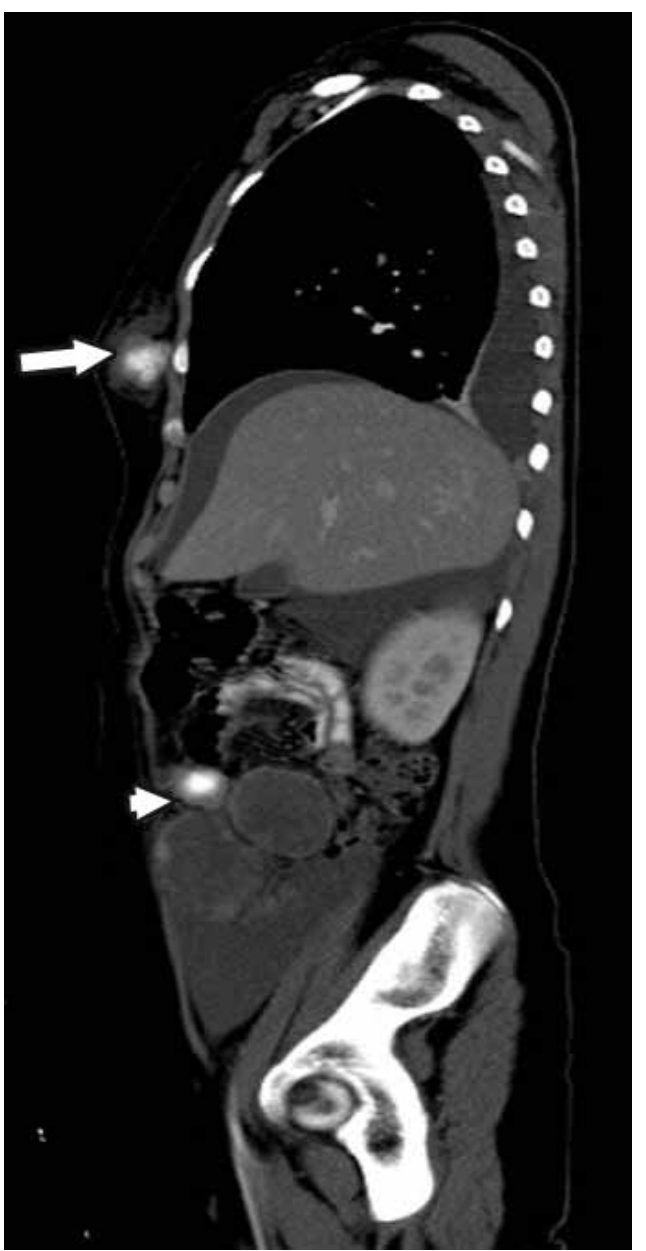

Fig. 4 Sagittal CT image shows calcified tumour deposits in the breast (arrow) and on the peritoneum (arrowhead). A lobulated noncalcified mesenteric metastasis inferior to the calcified peritoneal deposit can be seen.

with recurrent osteosarcoma carrying an extremely poor prognosis. The site of first recurrence affects the survival of patients with local or distant relapse. Patients with extrapulmonary metastases had significantly worse (9\%) five-year survival rates than patients with pulmonary metastases (19\%) and local recurrence (39\%). Additionally, patients with early recurrence or poor histological response to preoperative chemotherapy had poor overall survival.(2)

The skeleton is the most common site of extrapulmonary metastasis from osteosarcoma, ${ }^{(1,3)}$ and almost all bone metastases show high uptake on bone scan. On magnetic resonance (MR) imaging, these lesions exhibit low-signal intensity with dark foci (in keeping with calcification) and heterogeneous signal intensity on T1- and T2-weighted images, respectively, showing variable enhancement. Also, distant lymph node metastasis from osteosarcoma is not uncommon. Metastatic lymph nodes are typically rim-enhancing with central necrosis on CT and anechoic on ultrasonography. Conversely, metastases to the pancreas, liver, kidney and bowel are uncommon, and metastases to the peritoneum and subcutaneous tissue are extremely rare. Peritoneal metastases are typically well-defined and visualised as homogeneously hypodense masses on CT. Subcutaneous metastases are isointense and hyperintense on $\mathrm{T} 1$ - and $\mathrm{T} 2$-weighted images, respectively, and demonstrate avid homogeneous enhancement on MR imaging. The presence of calcification in metastatic lesions is strongly and positively correlated with uptake on bone scan. ${ }^{(1)}$

In a retrospective study over a ten-year period by Lookingbill et $\mathrm{al}_{,}{ }^{(4)} 420$ out of 4,020 patients $(10 \%)$ with metastatic disease had cutaneous metastases. In the majority of these patients $(72 \%)$, the first sign of extranodal metastatic disease was cutaneous metastases, some of which were found in the vicinity of the primary tumour, as well as at distant sites. Skin nodules were the most frequent presentation of cutaneous metastases, with inflammatory, cicatricial and bullous lesions also described in the study. The most common histopathology of cutaneous metastasis reported was adenocarcinoma, while the most common primary malignancies were breast carcinomas, malignant melanomas, and head and neck carcinomas. ${ }^{(4)}$

The breast is an uncommon site of metastatic disease, as metastasis to the breast from extramammary malignancy is very rare. In a recent study by Surov et al, ${ }^{(5)}$ the prevalence of breast metastasis was found to range from $0.12 \%$ to $4.92 \%$. The majority of these breast metastases were discovered incidentally during staging $\mathrm{CT}$ for a known primary tumour that was associated with other systemic metastases, as was the case in our patient. A small number of patients in the same study had metastasis to both breasts. On CT, the breast metastases appeared as circumscribed masses with marked or moderate enhancement. The most common mammographic patterns of breast metastases were solitary or multiple, round or oval masses with circumscribed margins of high or intermediate density. On ultrasonography, breast metastases were found to appear mostly as oval or round hypoechoic masses with circumscribed margins or microlobulations and posterior enhancement. On MR imaging, most lesions appeared as isointense to normal breast parenchyma on T2- and T1-weighted images, but showed homogeneous enhancement with types 2 or 3 kinetic curve. Associated primary malignancies in the study included malignant melanoma (31\%), and genital (18\%), lung (16\%), urologic (12\%) and gastrointestinal tumours $(7 \%)$. Sarcoma was reported to be the least common (4\%) primary tumour associated with this metastatic disease. ${ }^{(5)}$ Patients with breast metastasis were reported to have an overall mean survival of 22.4 months after diagnosis. ${ }^{(6)}$

Nonskeletal extrapulmonary metastases from osteosarcoma are uncommon, peritoneal metastases are extremely rare, while metastases to the skin and breast are even rarer. To our knowledge, breast metastasis from osteosarcoma has only been reported once, in a ten-year-old girl who developed breast metastases from chondroblastic osteosarcoma of the tibia three years after completion of treatment for pulmonary recurrence. ${ }^{(7)}$

In summary, our report describes the CT findings of multiple calcified and noncalcified tumour deposits in the lungs, pleura, peritoneum, chest wall and both breasts of a young patient who suffered from osteosarcoma relapse at 18 months from the time of diagnosis. 


\section{REFERENCES}

1. Kim SJ, Choi JA, Lee $\mathrm{SH}$, et al. Imaging findings of extrapulmonary metastases of osteosarcoma. Clin Imaging 2004; 28:291-300.

2. Gelderblom H, Jinks RC, Sydes $M$, et al. Survival after recurrent osteosarcoma: data from 3 European Osteosarcoma Intergroup (EOI) randomized controlled trials. Eur J Cancer 2011; 47:895-902.

3. Giuliano AE, Feig S, Eilber FR. Changing metastatic patterns of osteosarcoma. Cancer 1984; 54:2160-4.

4. Lookingbill DP, Spangler N, Helm KF. Cutaneous metastases in patients with metastatic carcinoma: a retrospective study of 4020 patients. J Am Acad Dermatol 1993; 29:228-36.

5. Surov A, Fiedler E, Holzhausen HJ, et al. Metastases to the breast from non-mammary malignancies: primary tumors, prevalence, clinical signs, and radiological features. Acad Radiol 2011; 18:565-74.

6. Vaughan A, Dietz JR, Moley JF, et al. Metastatic disease to the breast: the Washington University experience. World J Surg Oncol 2007; 5:74.

7. Roebuck DJ, Sato JK, Fahmy J. Breast metastasis in osteosarcoma. Australas Radiol 1999; 43:108-10. 